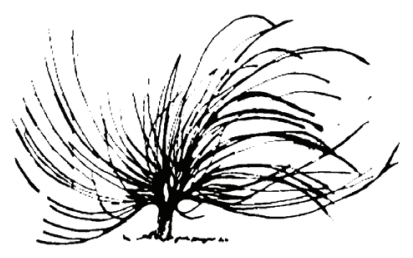

\title{
La metodología participativa para la intervención social: Reflexiones desde la práctica
}

\author{
Flor Abarca Alpizar ${ }^{1}$ \\ Universidad Nacional \\ Heredia, Costa Rica \\ fabarca@una.cr
}

\begin{abstract}
Resumen
La participación es una necesidad humana sentida y en conexión con el ser, tener y estar de las personas. Si promovemos la participación, fortalecemos las posibilidades de transformación, tanto a nivel individual como colectivo, generando niveles crecientes de autonomía e independencia para el mejoramiento integral de las condiciones de vida. La metodología participativa para la intervención social tiene estrecha relación con todos y cada uno de los momentos de los procesos de aprendizaje, garantiza su efectividad, unidad e integralidad para la realización de procesos de interaprendizaje de impacto real en la vida de las personas aprendientes. La ausencia de un planteamiento metodológico integral aplicado a los procesos de intervención social nos remite a las personas participantes al desgaste, al activismo, a la realización de procesos aislados y de poca efectividad y, en el peor
\end{abstract}

\section{$(1)(9)$}

Recibido: 9 de febrero de 2016-Aprobado: 25 de abril de 2016

1 Socióloga - Metodóloga. Doctora en Educación con énfasis en Mediación Pedagógica. Académica de la Universidad Nacional de Costa Rica en la Vicerrectoría de Docencia donde facilita procesos de formación y actualización metodológica y pedagógica con el profesorado universitario. Se ha desempeñado como consultora y asesora nacional e internacional y como profesora invitada en universidades del Ecuador, Nicaragua, Panamá y México. 
de los casos, a la inversión de recursos humanos, económicos y físicos en proyectos de poco aliento e impacto social. Si nos preocupamos solo por la transmisión de contenidos de manera agradable con el uso de lo último de "la tecnología educativa" o de técnicas o "dinámicas", sin un proceso metodológico integral y participativo, provocamos el conformismo, la pasividad y el silencio de las personas aprendientes, siendo precisamente con las que debemos lograr mayor apertura y confianza.

Palabras clave: Epistemología, holismo, interaprendizajes, metodología, participación.

\begin{abstract}
Participation is a strong human need resulting from people's sense of being, having, and taking a place in the world. By promoting participation, we strengthen our transformation possibilities, both at a personal as well as at a collective level, generating growing levels of autonomy and independency to comprehensively improve life conditions. Participatory methodology for social intervention is closely related to all learning-process moments and it guarantees their effectiveness, unity, and completeness to carry out shared learning processes with real impact on the life of learners. The absence of a comprehensive methodological formulation leads us to exhaustion, to activism, to perform isolated and poorly effective processes and, in the worst case scenario, to invest human, economic and physical resources in projects of disincentive or low social impact. If we focus our concern only on conveying content in a pleasant way using the latest "educational technology" or techniques or "dynamics" without a comprehensive and participatory methodological process, we are promoting conformism, passiveness, and silence of learners, when they are exactly the individuals with whom we must achieve openness and confidence in a higher degree.
\end{abstract}

Keywords: epistemology, holism, shared learning, methodology, participation 


\section{Introducción}

Este ensayo es producto de las reflexiones críticas sobre las experiencias de facilitación de múltiples talleres sobre metodología participativa con profesionales en docencia universitaria, promoción social e intervención social participativa, a nivel nacional y latinoamericano. Estas conceptualizaciones teóricas surgen de la sistematización de experiencias (Jara, 2012, p. 163):

Entendemos por sistematización de experiencias a la interpretación reflexiva y crítica de una o varias experiencias, que, a partir de su ordenamiento y reconstrucción, descubre o explícita el proceso vivido, los factores que han intervenido en dicho proceso, cómo se han relacionado entre sí, y por qué lo han hecho de ese modo. La sistematización es un ejercicio que se realiza en torno a experiencias prácticas que se proponen cambiar una realidad social.

En la práctica de asesoría y acompañamiento a procesos de intervención social, hemos constatado el uso de métodos, técnicas, procedimientos e instrumentos participativos en sustitución de la concepción metodológica participativa. Esto sucede porque los métodos y las técnicas son concretas, medibles y constatables, mientras que la concepción epistemológica de la metodología participativa nos remite al intercambio de seres y saberes desde aspectos sensitivos, subjetivos y afectivos, es decir, desde las emociones y los sentimientos que no son visibles ni cuantificables.

La sociedad actual pone el acento en lo cuantitativo, lo concreto y medible, a la transmisión mecánico - lineal de saberes estandarizados entre personas separadas, basada en el control, que imposibilita el encuentro, la creatividad y la conectividad entre los seres vivos. De esta forma, la cultura occidental invisibiliza los sistemas, los procesos, los sentimientos y la diversidad de los contextos, al imponer una mirada basada en modelos y patrones aceptados acríticamente, como resultado de un modo mecanicista, estrecho, fijo y rígido de pensar y actuar. 


\section{¿Cómo conocemos y cómo aprendemos? Visiones epistemológicas}

Las visiones de mundo o cosmovisiones que asumimos como humanidad están sustentadas en concepciones epistemológicas o teorías del conocimiento. En los últimos 400 años, la humanidad ha estado bajo la influencia de la visión de mundo o paradigma mecanicista-cartesiano, cuyas principales tendencias son la visión fragmentada, la racionalidad, la linealidad, énfasis en lo cuantitativo y medible, el positivismo y el abuso del método científico. Al respecto, Capra (1994, p. 44) plantea:

Hoy se ha vuelto evidente que el excesivo énfasis puesto en el método científico y en el pensamiento analítico y racional ha provocado una serie de actitudes antiecológicas. La perspectiva cartesiana del mundo es mecanicista. El mundo ya no puede percibirse como una máquina formada por una gran cantidad de objetos, sino que ha de concebirse como una unidad indivisible y dinámica cuyos elementos están estrechamente vinculados.

En la cultura occidental prevalecen las concepciones epistemológicas o visiones de mundo dicotómicas y centradas en el control, la intolerancia a lo diverso, el poder de dominio y control, la percepción de la vida separada de la Madre Tierra, de la naturaleza y de los seres vivos. Esta visión transversa todas nuestras acciones: la vida cotidiana familiar, laboral, la educación, la investigación, la salud, la economía, la política. Najmanovich (2014, p. 5) expresa, al respecto:

Nuestra cultura ha abordado los conflictos desde una posición dicotómica que nos lleva al temor de lo diverso y a tratar de controlarlo. En la modernidad, se impuso el modelo disciplinario en la educación, el acento está puesto en la enseñanza entendida como transmisión mecánico-lineal de saberes estandarizados entre individuos separados. Los afectos, en especial el miedo, han sido cruciales en la consolidación de la sociedad disciplinaria y así también la confianza y la alegría han de serlo en la construcción de una ética del encuentro para afrontar los desafíos contemporáneos. En la actualidad vivimos tiempos agitados, nuevas formas de control están creciendo y también otros modos de pensar, habitar y convivir desde la ética del encuentro. Los abordajes de la 
complejidad nos permiten comprender el valor productivo de los antagonismos y nos ayudan a convivir con la tensión inherente a la vida. En la educación el énfasis está puesto en los aprendizajes como modo de existencia del ser vivo profundamente conectado con su ambiente.

Necesitamos construir la ética del encuentro en nuestras relaciones con la familia, en la universidad, en la comunidad, con la naturaleza y los demás seres vivos, con lo que sentimos, pensamos y hacemos. Esta ética del encuentro, basada en comunidades aprendientes, redes y conectividades, demanda nuevas formas interdisciplinarias para construir aprendizajes integrados con los sistemas vitales, porque todo ser vivo, incluidas las personas, necesitan de los aprendizajes para vivir, lo cual demanda nuevos enfoques epistemológicos integrales e interdisciplinarios.

\section{La epistemología como teoría del conocimiento}

Todos éramos humanos hasta que: la raza nos desconectó, la religión nos separó, la política nos dividió y el dinero nos clasificó.

Autoría anónima

En pleno siglo XXI de nuestra era, las metodologías vigentes para la intervención social, ya sean estas funcionalistas, positivistas o científicas, conductistas o constructivistas, se inspiran en concepciones epistemológicas sustentadas en el paradigma dominante positivista, mecanicista, newtoniano - cartesiano, que fragmenta, separa y aísla los fenómenos que corresponden a objetos de estudio con visiones disciplinares, fragmentadas, lineales y dicotómicas, provocando serias distorsiones con los procesos vitales caracterizados por sus dinámicas y relaciones interdependientes, diversas, integrales, interdisciplinarias, complementarias y complejas. Hoy se sabe, por la nueva cosmología, que los seres vivos poseen no solamente masa y energía. Son portadores también de información, historia, complejidad, crean órdenes que comportan un cierto nivel de subjetividad; Assmann (2002, p. 27) lo plantea así: 
Los procesos cognitivos y vitales descubren su lugar de encuentro marcado desde siempre, en el centro de lo que es la vida, como proceso de autoorganización, desde el plano biofísico hasta el de los ámbitos sociales, la vida quiere seguir siendo vida, la vida que se gusta y que se ama y anhela ampliarse en más vida. La producción y reproducción biológica y social de la vida no se deja encuadrar en esquemas econométricos, porque los seres vivos entrelazan necesidades y deseos de un modo mucho más complejo. Ambos forman un tema unificado.

De acuerdo con Assmann (2002), los procesos vitales y los procesos de aprendizaje son la misma cosa. Estar en proceso de aprendizaje significa conectarnos con los sentidos vitales, como lo hacen todos los seres vivos, en forma flexible y adaptativa, para integrarnos con la dinámica de seguir aprendiendo, es decir, de seguir viviendo.

\section{Uso y abuso del positivismo metodológico}

Como hemos afirmado, las concepciones epistemológicas de las metodologías más utilizadas en procesos de intervención social se sustentan en el paradigma dominante:

... la epistemología positivista redujo la multifacética, dinámica y contextualizada actividad de conocer hasta aplastarla y convertirla en un producto: el objeto de conocimiento... y el ser humano real como todo ser vivo, que jamás podría existir ni actuar aisladamente, fue concebido como un individuo-sujeto absolutamente independiente... (Najmanovich, 2014, p. 10)

Las metodologías no sean neutrales, todas llevan implícita y responden a la visión de mundo y formas de entender la vida para las que fueron creadas. En el paradigma newtoniano-cartesiano vigente encontramos que las metodologías funcionalistas o positivistas más usadas promueven relaciones fragmentadas, lineales, cuantitativas, racionales entre la humanidad, la naturaleza y los otros seres vivos. 


\section{La metodología participativa como proceso y complejidad}

El surgimiento de la concepción metodológica participativa y procesual responde al pensamiento complejo, que propone nuevas miradas al conocimiento de la realidad, de la vida, de las relaciones entre las personas, de los seres vivos y de la naturaleza, como interdependientes, integrales, complejas y autoorganizadas. Es una alternativa al predominio cientificista y positivista. Najmanovich (2008, p. 175), describe el surgimiento del pensamiento complejo:

Las concepciones positivistas, que han reinado desde los inicios de la modernidad hasta mediados del siglo XX hoy están en franca declinación. El mundo estable al que aspiraron y que, con relativo éxito pudieron construir, ha entrado a una fase de transformaciones que ha tomado proporciones imposibles de soslayar. El universo mecánico, lineal y predecible se está desmoronando... abandonando la idea del conocimiento como un mundo independiente del observador. El pensamiento complejo no admite las separaciones absolutas, ni los sistemas aislados de la ciencia clásica. Por eso exige un cambio en el tratamiento global del conocimiento tanto a nivel conceptual como de las prácticas institucionales.

Los fundamentos epistemológicos de la metodología participativa tienen estrecha relación con todos y cada uno de los momentos de los procesos de intervención social, garantizando, entre otros aspectos, la efectividad del trabajo, su unidad e integralidad para la realización de procesos de impacto real en la vida de las personas. La ausencia de un planteamiento metodológico participativo e integral nos remite al desgaste, al activismo, a la realización de procesos aislados y de poca efectividad y, en el peor de los casos, a la inversión de recursos humanos, económicos y físicos en proyectos de poco aliento e impacto social.

Todo proceso es el resultado de la interacción y cooperación de un grupo de personas que actúan subjetivamente dentro de las exigencias derivadas de las necesidades que desea solucionar. Este proceso tiende a ser complejo y dinámico, como nos lo explica Najmanovich (2015, N50): 
En las últimas décadas se ha gestado una gran transformación de nuestra forma de concebir el conocimiento. Lejos de los planteos positivistas, dualistas y disciplinarios, diversos enfoques han ido creciendo y entramándose en un diálogo fértil y productivo y dando lugar a la multidimensionalidad y complejidad de la experiencia humana. Es importante que exploremos el conocimiento teniendo en cuenta los aportes actuales del pensamiento complejo, la biología del conocimiento, las neurociencias, la psicología cognitiva y la filosofía. Porque no estamos viviendo un mero cambio de modelos, teorías y paradigmas, sino una profunda mutación de nuestras formas de conocer.

Al cambiar de enfoque, al aplicar nuevas y desafiantes maneras de construir conocimientos desde la complejidad, al pasar del pensamiento basado en la fragmentación a la integralidad y su complejidad, constatamos que las interacciones subjetivas constituyen fuerzas poderosas capaces de movilizar, en cada una de las personas integrantes del proceso, lo mejor de su sensibilidad, imaginación, voluntad, creatividad, solidaridad, inventiva, afectividad, empatía, respeto y demás recursos personales, generando así una fuerza integradora que va de lo individual a lo grupal, transformando los recursos internos de las personas en catalizadores de energía social transformadora. Es, precisamente, este caudal sinérgico de los recursos no convencionales lo que hace del proceso metodológico un importante espacio sinérgico de interaprendizajes.

La metodología participativa pretende descifrar desde el interior de los procesos de intervención social su sentido, interviniendo activa y conscientemente en su transformación, haciendo de las actividades espontáneas de las personas un proceso consciente. La metodología participativa implica esfuerzos permanentes y sistemáticos de análisis, estudio, reflexión sobre la realidad, los proyectos, programas y prácticas en las que estamos trabajando. En estos tiempos agitados en que la crisis, el cambio y la complejidad son la norma y no la excepción, Najmanovich (2014, p. 12), plantea:

Se impone dejar de lado los modelos predeterminados y universales para construir un conocimiento vivo, situado, multidimensional y complejo. Un saber que no quede atrapado en un conjunto de definiciones a repetir, sino que pueda aportarnos herramientas 
para pensar, entendiendo que honrar la complejidad no significa que podamos abarcarla, sino que somos conscientes de que todo conocimiento humano es necesariamente limitado, sesgado y variable. Los escenarios no pretenden ser representaciones de la realidad y descripciones completas, sino dispositivos para pensar que siempre están abiertos a la novedad y la transformación.

Se necesita, entonces, de la participación, la libertad, la creatividad para construir nuevos escenarios abiertos, hacia el encuentro de seres y saberes.

\section{¿Cómo ver lo invisible?}

... Solo se ve bien con el corazón. Lo esencial es invisible para los ojos... El Principito, Antoine de Saint Exupéry

La metodología participativa nos invita a dar nuevas miradas, visibiliza aspectos tradicionalmente ocultos como las interculturalidades, las cosmovisiones, la vida cotidiana, las subjetividades, las percepciones e intuiciones, los aprendizajes, la historiografía, la equidad, la diversidad entre las personas, entre otras.

Si la vida está constituida por procesos, sistemas, ondas, fluctuaciones, rizos, complejidad y caos, necesitamos aplicar a los procesos de intervención social una concepción epistemológica y metodológica coherente con todo ello, con la vida misma, para poder lograr aprendizajes verdaderos, duraderos, para la vida toda, como lo afirma Assmann (2002, p. 75):

Los proyectos de aprender y de conocer vuelven a hacer las pases con el carácter más bello y más olvidado de la finitud: el de ser ondas, flujo, dinámica, autoorganización. Recuperar esta visión finita y dinámica de la vida y del conocimiento implica aceptar que sus procesos autoorganizativos nunca pueden superar los límites de la finitud de los nichos vitales que nos albergan.

Así entendida, la metodología participativa se fundamenta en los aspectos epistemológicos que hacen referencia a la concepción integral 
de la vida, el cosmos, la naturaleza, las personas y demás seres vivos como interdependientes, diversas, históricas, cambiantes y contradictorias, para conocer la realidad y actuar en ella en forma integral, coherente, participativa y colocando a la vida, a los seres vivos, a la naturaleza, a la humanidad como los elementos centrales de todas las acciones. En este sentido, Leis (1989, p. 31), explica:

En la metodología participativa..., el saber es un proceso vivo, dinámico, que se desarrolla en la interacción entre las personas, en su reflexión compartida sobre lo que hacen, lo que buscan, lo que aspirar y desean. Los conocimientos deben producirse en el propio proceso educativo. Esta producción no es individual, sino colectiva, a través del intercambio, el diálogo y la reflexión, relacionando la práctica y la teoría.

La metodología participativa no se ve; se siente y se respira, se percibe a través de los sentidos que usan las personas participantes en los procesos en los cuales se utiliza, promoviendo espacios con utilidad, intencionalidad, integralidad y sentido para las personas participantes.

\section{¿Cómo se aplica la metodología participativa?}

La metodología participativa es una concepción alternativa al positivismo dominante para construir procesos de intervención social colectivos, autónomos, solidarios, respetuosos y participativos. Los principios y fundamentos epistemológicos, filosóficos y conceptuales que la sustentan se han aplicado en espacios de docencia, investigación y extensión universitaria y en procesos de educación comunitaria no formal con poblaciones excluidas y diversas como mujeres, poblaciones autóctonas, campesinado, ecologistas y otros por medio de:

a. Las dimensiones de la metodología participativa.

b. El bucle o rizo metodológico práctica - teoría - práctica.

c. Aplicación del bucle P-T-P a los métodos y técnicas participativas. 


\section{a) Las dimensiones de la metodología participativa}

El proceso metodológico participativo está integrado por cuatro dimensiones interrelacionadas, como lo explica Jara (1987, p. 45):

La dimensión comunicativa, la dimensión pedagógica, la dimensión investigativa y la dimensión creadora o la producción teórica. Estas cuatro dimensiones están presentes en cualquier acción de intervención que realicemos y va a depender de las particularidades de las personas participantes, el contexto en que actúan, los objetivos que perseguimos, el proceso que se quiere potenciar, que intencionamos o priorizamos una, varias o todas sus dimensiones.

No pueden separarse en momentos distintos: no existe un momento para investigar, otro para aprender, otro para comunicarse y otro para crear producción teórica. Todos los procesos anteriores se dan de manera conjunta en un solo proceso integral, práctico y conceptual, a través de diferentes acciones, en las que a veces el peso particular de una de las dimensiones puede ser mayor que otras, pero siempre están presentes todas.

\section{La dimensión investigativa de la metodología participativa}

En la medida en que se necesita conocer permanentemente la realidad que se quiere intervenir en todos sus aspectos: económicos, sociales, políticos, ideológicos, culturales, ecológicos, salud y vida, tanto en la realidad inmediata que viven las personas, como en sus relaciones con la realidad histórica y social global, es necesario fortalecer los procesos investigativos para transformar las condiciones de vida de las personas.

Esta dimensión está constantemente presente en los procesos de aprendizaje universitarios, educación secundaria, primaria y comunitaria no formal, y tiene como uno de sus momentos culminantes el proceso de conocimiento, de búsqueda y recuperación de la memoria colectiva, de los hallazgos contextuales y las formas particulares como las personas sienten, perciben e interpretan determinados fenómenos, así como la búsqueda de diversas fuentes y estudios especializados existentes para profundizar las discusiones y elaboraciones teóricas en el proceso de aprendizaje. 


\section{La dimensión pedagógica de la metodología participativa}

La metodología participativa se inscribe en procesos que requieren de la promoción de los aprendizajes universitarios, educación formal y no formal comunitaria, articulando el conocimiento existente con los nuevos conocimientos, avanzando a niveles cada vez más profundos. Para ello requerimos de la intencionalidad de la dimensión pedagógica para fortalecer los interaprendizajes.

Esta dimensión pedagógica está constantemente presente para orientar el tránsito ordenado y sucesivo de conocimientos sobre fenómenos sociales o naturales, hacia la comprensión teórica global de esos procesos, vinculados permanentemente con la vida en su totalidad, porque aprender es vivir y vivir es aprender. No hay vida posible sin aprendizajes para todos los seres vivos de este planeta, de los cuales la humanidad forma parte.

\section{La dimensión comunicativa de la metodología participativa}

La metodología participativa fortalece procesos realizados colectivamente y en función de acciones con carácter colectivo y de impacto social en docencia, investigación y extensión universitaria, así como también en la educación formal institucionalizada.

La dimensión comunicativa está constantemente presente en estos procesos para permitir el diálogo creador, intercambio de conocimientos, reflexiones, opiniones y conclusiones; los interaprendizajes y el enriquecimiento permanente del propio saber con el de otras personas, porque involucra activamente la participación de todas las personas en bucles de realimentación permanentes.

\section{La dimensión creadora de la metodología participativa: Los productos}

Desde la integralidad de las dimensiones de la metodología participativa, la expresión creadora se considera como algo concomitante al proceso y no como el producto final. Esta concomitancia se manifiesta por actitudes y capacidades que se potencian por medio de acciones variadas y permanentes. Se da en el momento mismo en que las personas logran generar acciones creadoras que dinamizan y retroalimentan el proceso. 
Su mayor reto es lograr que las personas aprendientes en interacción se construyan como sujetos por medio de la expresión creadora que trasciende y realimenta al mismo tiempo. La expresión creadora cristaliza la realización personal con resultados muy concretos. Las personas se realizan en la recreación de la realidad y con esta. El sello de la personalidad tiene que ser observable a lo largo de todo el proceso, de esta forma se pone en práctica una gran dosis de imaginación creadora.

\section{b) La relación entre práctica - teoría - práctica: El bucle de la me- todología participativa}

La relación integral e interdependiente entre práctica - teoría práctica surge de la concepción metodológica dialéctica de la educación popular (elaborada por la Red Mesoamericana de Educación Popular Alforja), a la cual en este texto denominamos bucle metodológico participativo y procesual.

Realizar procesos participativos, ya sean investigativos, comunicativos o de interaprendizaje, significa darles unidad, coherencia, articulación e integralidad, aplicando el bucle que integra la relación entre práctica - teoría- práctica, en procesos de ida y vuelta y en espiral ascendente. Este bucle se aplica a los ejes temáticos propuestos en el plan de estudio o contenidos por medio de diversidad de formas creativas como métodos, técnicas y procedimientos para la promoción integral de los procesos de aprendizaje, como lo explica Leis (1989, p. 45):

La relación entre práctica -teoría -práctica tiene su fundamento en el hecho de que la práctica social es fuente, criterio de verdad y fin último del proceso de conocimiento. La práctica social es el conjunto de actividades que realizamos conscientemente e intencionadamente en lo económico, lo político, lo ideológico, lo cultural, lo cotidiano; esta práctica social está inmersa en la realidad. La reflexión que hagamos sobre esta práctica y esta realidad no pueden ser ideas abstractas, sino por el contrario, elementos que deben confrontarse continuamente con la realidad y la práctica. La finalidad de todo este proceso de conocimiento es la transformación de la realidad, la superación de la práctica social haciéndola más eficaz y eficiente en función de la liberación del pueblo. 
El bucle de la metodología participativa es la relación integral entre práctica - teoría - práctica. Se aplica en procesos educativos universitarios, investigativos y de extensión comunitaria para la promoción integral de los aprendizajes. ${ }^{2}$ Por medio de la adaptación y síntesis compartimos los momentos del proceso integral práctica - teoría - práctica.

\section{Primer momento del bucle metodológico: Lo que sabemos y sentimos}

Aplicar el primer momento del bucle práctica - teoría - práctica significa partir de la vida cotidiana, del contexto, las problemáticas, los saberes, los sentimientos, las experiencias, sentidos, percepciones y emociones de las personas participantes y se hace realidad, entre otras, por medio de:

La valoración de las personas participantes: Las personas aprendientes son las protagonistas de los procesos participativos, son las que determinan el proceso, lo llenan de sentidos y significados y le dan su propia dinámica.

El reconocimiento de los problemas en la realidad y el contexto de las personas aprendientes: significa conocer la realidad en que viven las personas y adecuarnos flexiblemente a cada particularidad, de manera que el aquí y el ahora sean, permanentemente, el punto de partida. Recordemos que el contexto también educa.

El respeto a la individualidad y los sentimientos de las personas aprendientes: hacer esfuerzos para fortalecer la autoestima y valoración personal de cada quien, tomando en cuenta en todo momento a las personas y su individualidad.

El respeto por las diversidades: todas las personas conformamos la humanidad y somos interdependientes con los otros seres vivos que habitamos en este hogar común llamado planeta Tierra. Las diferencias son producto de las interculturalidades y de las diferencias impuestas por condiciones de raza, etáreas, sociales, económicas, ideológicas políticas, religiosas, culturales, étnicas y de género.

2 Los momentos del bucle de la metodología participativa: Práctica - teoría - práctica corresponden a la adaptación y síntesis realizada con base en Abarca, 2012 (pp. 80 - 84). 


\section{Segundo momento del bucle metodológico: Teorización}

La construcción de conocimientos colectivos o la teorización colectiva es el proceso que se promueve en este segundo momento del bucle o proceso metodológico participativo; precedido del anterior, promueve la reflexión, la conceptualización y la construcción colectiva de conocimientos, entre otras, por medio de acciones que:

Impulsen el trabajo colectivo y en equipo: el trabajo colectivo y grupal es la forma natural e idónea para producir conocimientos colectivamente. Para lograrlo es necesaria la disposición a este, el convencimiento de su efectividad, el respeto a las ideas y habilidades de las otras personas, la humildad para aceptar sugerencias y reconocer errores, una actitud fraterna y solidaria, un clima de alegría y motivación.

Promuevan la comunicación, el diálogo y la participación: dentro de las relaciones humanas fundamentales, la comunicación encabeza la lista de posibilidades para lograr relaciones basadas en el respeto, la tolerancia y el enriquecimiento. Por la comunicación se hace posible la actualización y el enriquecimiento de las personas. La comunicación da sentido a la vida humana y, tanto más rica será la vida, cuanto más y mejor puedan las personas comunicarse, cuanto más y mejor puedan darse y compartirse en diálogos de seres y saberes.

Fortalezcan la alegría y la creatividad: es importante recuperar en los procesos participativos la alegría y creatividad abriendo espacios de encuentro y amistad, que dé gusto llegar a ellos, en donde la alegría y la creatividad no tengan límites. Es necesario romper con los espacios y actividades aburridas, tediosas, cansadoras y pesimistas, que implican tareas o "cargas" de las múltiples que ya tenemos las personas, porque aprendemos en clave gozo.

\section{Tercer momento del bucle metodológico: Aplicación práctica}

Este tercer momento del bucle o proceso metodológico participativo, precedido de los dos anteriores, promueve la aplicación de las reflexiones y conceptualizaciones colectivas a la práctica, a los diversivos contextos, a la vida cotidiana. La vuelta a la práctica es el momento para la aplicación de los nuevos conocimientos, realizando acciones, entre otras, como: 
La elaboración de propuestas para mejorar la calidad de vida: en los procesos de extensión universitaria y educación comunitaria encontramos que es en el espacio cotidiano donde con mayor fuerza cobran sentido y significación los problemas, las necesidades, las carencias, y vacíos económicos, políticos, ideológicos y de diversa índole, así como las alegrías y esperanzas. Las propuestas para mejorar la calidad de vida se construyen integralmente desde la vida cotidiana para que tengan sentido de pertenencia, significación, utilidad, y logren proyectarse a las personas y la sociedad en general.

La construcción de espacios para reflexionar, cuestionar, defender y negociar: significa proponer momentos y espacios para el análisis y la reflexión de la situación de cada persona, cada comunidad, país y región, para generar la criticidad y el cuestionamiento sustentado, que permitan acciones de propuesta y negociación acordes a los problemas y necesidades de las personas participantes.

\section{La integralidad entre la práctica - la teoría y la práctica}

El proceso integral entre la práctica - teoría - práctica representa el bucle de la metodología participativa y su aplicación es fundamental en los procesos de aprendizaje, investigativos y comunicativos para el logro de su efectividad e impacto. Su inserción en los métodos, técnicas e instrumentos es garante de su efectividad, en los procesos de construcción colectiva. Sintetizando lo anterior, Leis $(1989$, p. 27) reafirma sobre la relación práctica - teoría - práctica:

Cuanto más sencillo, próximo y cercano sea el punto de partida y cuanto más se relacione con la realidad, mayores son las posibilidades de comprensión y conocimiento... Partir de la práctica no significa quedarse en las apariencias, sino ir acercándose a la esencia de esa realidad...La teorización debe incorporar los conocimientos de otras personas, de otras épocas, de la historia, no en forma mecánica, porque no se trata de transmitir conocimientos, sino producirlos con los aportes de nuestra propia reflexión... Volvemos a la práctica, pero esto no significa que volvamos al mismo punto de partida, sino más bien a la posibilidad de una nueva práctica, mejorada, para transformar mejor la sociedad, una práctica cada vez más transformadora. 


\section{c) La aplicación del bucle de la metodología participativa P-T-P a los métodos y las técnicas participativas}

Para la aplicación de la metodología participativa a los métodos y las técnicas, se les intenciona o enfoca la dimensión correspondiente, ya sea comunicativa, investigativa, pedagógica y creativa, de acuerdo con los proceso de intervención social en ejecución. También se intenciona, al método o técnica respectiva, el bucle de la metodología participativa, a saber, la relación entre práctica - teoría - práctica.

Existen muchos métodos participativos, así como bibliografía e información en internet sobre estos, pero poca veces se indica que necesitamos intencionar y enfocar su uso, porque no es lo mismo emplear un "diagnóstico participativo", una "historia de vida", una "entrevista en profundidad" para una investigación, un evento educativo, un proyecto de extensión universitaria o una sistematización de experiencias. Cada dimensión le da su uso y sentido particular.

La aplicación del bucle de la metodología participativa práctica - teoría - práctica permite la integralidad al intencionar el sentir, pensar, las percepciones e intuiciones en un primer momento, para dar espacio a la reflexión crítica, el análisis y la construcción colectiva de conocimientos, para realizar aplicaciones prácticas a la vida cotidiana, a la realidad y contexto.

A continuación se comparte un ejemplo sobre la aplicación de la metodología participativa al método taller.

\section{El taller: Método participativo por excelencia ${ }^{3}$}

El auge de la metodología participativa, producto de la sistematización de los procesos de educación popular participativa en América Latina, ha permeado todas las instancias educativas en nuestro continente y con impacto también en Europa, especialmente en los Países Nórdicos y España. Los talleres surgen como las primeras alternativas de aprendizaje participativo y democrático, frente al método tradicional de transmisión de conocimientos "de una persona que sabe a otra que no...". Se origina en los procesos de educación popular en América Latina, en la segunda mitad del siglo XX. Estos procesos exitosos de las comunidades de aprendizaje no formales y alternativas animan y

3 Adaptación con base en Abarca (1999) 
motivan a los sistemas estatales y gubernamentales a asumir la metodología participativa y al método taller, como impulsores de los interaprendizajes, ampliamente validados. Hoy nos encontramos con su uso en instituciones educativas formales y en la academia universitaria por su versatilidad, flexibilidad, propuesta creativa y organizativa.

\section{El taller como intercambio de seres y saberes}

En los talleres, como espacios de encuentro y de intercambio de saberes entre personas aprendientes, las subjetividades, las percepciones, intuiciones, vivencias y saberes, cobran importancia y relevancia. Es un método participativo muy útil para compartir aprendizajes, experiencias y vivencias individuales y colectivas. Su uso se ha ampliado como procesos de construcción colectiva de conocimientos, en donde las personas participantes son guiadas por propósitos comunes y el deseo de alcanzarlos en un tiempo determinado, que generalmente es corto, para construir situaciones de aprendizaje y de producción orientadas a conseguir metas comunes.

\section{La facilitación pedagógica de los talleres}

La facilitación de talleres conlleva el rompimiento de las tradicionales relaciones de dominio, control y poder presentes en los espacios educativos tradicionales. Esta ruptura epistemológica implica grandes retos y responsabilidades para las personas facilitadoras y participantes. La facilitación de talleres promueve relaciones respetuosas, solidarias, inclusivas y fraternales, para expresar las propias ideas, experiencias y sentimientos, sin acaparar el tiempo y el espacio del taller.

Por tanto, la facilitación toma posiciones, opciones vitales, manifiesta sus concepciones de vida, sus principios y valores éticos, así como los desacuerdos y divergencias ante hechos y situaciones de la realidad. Promueve la reflexión crítica y propositiva y no la creación de falsos consensos. También le corresponde tomar decisiones para la buena marcha de los procesos de aprendizaje, promoviendo el respeto y la tolerancia, la comunicación fluida y la motivación permanente y creativa hacia la participación y el intercambio de seres y saberes. 


\section{¿Cómo aplicamos la metodología participativa al método taller? La planificación de talleres participativos ${ }^{4}$}

Para la planificación o diseño de los talleres participativos se utiliza el instrumento denominado Diseño metodológico o cuadro guía. Este instrumento sirve para no improvisar y permite saber, con antelación, qué hacer, con quiénes, en cuánto tiempo, cómo hacerlo y qué se necesita para asegurar los interaprendizajes, la apropiación temática y el éxito de la actividad. Veamos la tabla 1, correspondiente al diseño metodológico o cuadro guía.

Tabla 1. La matriz del diseño metodológico

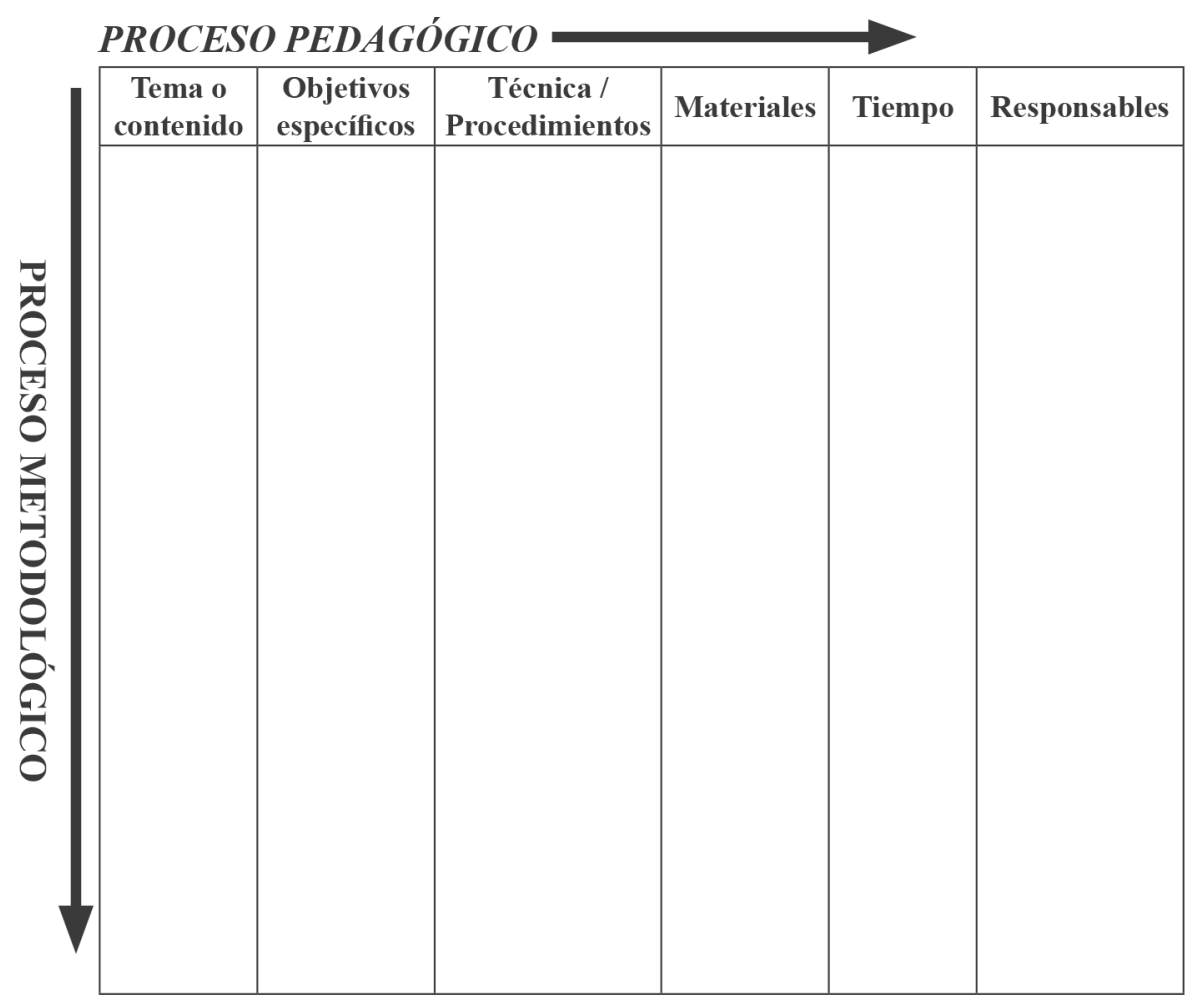

Nota: Elaboración propia.

4 Elaborado por medio de la revisión y actualización con base en Abarca y Candanedo (1992). 


\section{El proceso metodológico}

El proceso metodológico está presente en todo el diseño metodológico, dándole coherencia, unidad e integralidad al proceso educativo a desarrollarse. Se aplica a través del bucle de la metodológica participativa: práctica - teoría - práctica.

En la tabla 2 ejemplificamos la aplicación de la relación práctica - teoría - práctica en la planificación de un taller:

Tabla 2. La aplicación de la metodología participativa al método taller

\begin{tabular}{|c|c|c|c|c|}
\hline Contenidos & Objetivos específicos & Procedimientos & Materiales & Tiempo \\
\hline $\begin{array}{l}\text { Primer momento: } \\
\text { Partir de la práctica } \\
\text { Lo que sabemos y } \\
\text { sentimos: partimos } \\
\text { de la vida cotidiana, } \\
\text { las prácticas, las } \\
\text { experiencias, las } \\
\text { percepciones, } \\
\text { intuiciones, saberes, } \\
\text { otros. }\end{array}$ & $\begin{array}{l}\text { Colocamos acá el } \\
\text { primer objetivo } \\
\text { específico elaborado } \\
\text { en el encabezado o } \\
\text { portada del diseño } \\
\text { metodológico, } \\
\text { relacionado } \\
\text { con él. Partir de } \\
\text { experiencias, } \\
\text { prácticas, vivencias, } \\
\text { intercambio de seres } \\
\text { y saberes. }\end{array}$ & & & \\
\hline $\begin{array}{l}\text { Segundo momento: } \\
\text { Teorización } \\
\text { Reflexionamos y } \\
\text { profundizamos para } \\
\text { la construcción } \\
\text { colectiva de } \\
\text { conocimientos y la } \\
\text { teorización } \\
\end{array}$ & $\begin{array}{l}\text { Colocamos } \\
\text { acá el segundo } \\
\text { objetivo específico } \\
\text { relacionado con la } \\
\text { reflexión teórica } \\
\text { y la construcción } \\
\text { colectiva de } \\
\text { conocimientos. } \\
\end{array}$ & & & \\
\hline $\begin{array}{l}\text { Tercer momento: } \\
\text { vuelta a la práctica. } \\
\text { Aplicamos } \\
\text { conocimiento: } \\
\text { aplicamos } \\
\text { conocimientos a } \\
\text { la vida cotidiana } \\
\text { para la elaboración } \\
\text { de propuestas } \\
\text { transformadoras. }\end{array}$ & $\begin{array}{l}\text { Colocamos acá } \\
\text { el tercer objetivo } \\
\text { específico } \\
\text { relacionado con la } \\
\text { aplicación práctica } \\
\text { para mejorar las } \\
\text { condiciones de vida. }\end{array}$ & & & \\
\hline
\end{tabular}

Nota: Elaboración propia. 
De acuerdo con las tablas 1 y 2, la metodología participativa está en la totalidad del proceso de intervención social, es la que lo integra, le da sentido y cohesión. Esta intencionalidad se inserta en la redacción de los objetivos específicos, de forma sintética, uno por cada momento del bucle metodológico: práctica - teoría- práctica. A partir del objetivo específico, se anota el nombre al contenido y se describen los procedimientos.

\section{El cambio y nuestras incertidumbres cotidianas}

No se puede resolver un problema desde el mismo estado de conciencia que lo provocó. Albert Einstein

Como hemos afirmado, la participación es una necesidad humana y entre otros aspectos, la clave del éxito de los procesos de intervención social. La participación no se obtiene por decreto o buenas voluntades, necesitamos promoverla e incentivarla como responsabilidad ética hacia la transformación individual y colectiva.

La participación tampoco se logra con las viejas formas positivistas, dicotómicas, fragmentadas y disciplinares de abordaje. Exige un nuevo enfoque integral, desde la ética del encuentro y la interdisciplinariedad, donde el diálogo de seres y saberes, la autorganización y la autonomía son su intencionalidad permanente.

La metodología participativa se fundamenta en la concepción epistemológica holista y de la complejidad, y se aplica en conectividad con todos los momentos de los procesos de intervención social investigativos, de extensión, docencia universitaria y espacios de educación comunitaria. Garantiza la efectividad, unidad e integralidad de estos mismos y el impacto real en la vida de las personas aprendientes. Nos invita a realizar cambios en nuestra manera tradicional de hacer las cosas, dando énfasis a los aspectos cualitativos, afectivos, emocionales, interculturales y al diálogo de saberes. Se aplica en los procesos de intervención social por medio de: 
Las dimensiones de la metodología participativa: investigativa, comunicativa, educativa y creativa.

La relación integral práctica - teoría - práctica o bucle de la metodología participativa.

La aplicación del bucle de la metodología participativa P-T-P a los métodos y

las técnicas participativas, instrumentos y procedimientos

Para pasar de las formas tradicionales de abordaje a procesos integrales necesitamos hacer rupturas epistemológicas, reconociendo que todo cambio que implique movilizar nuestros saberes, nuestras creencias, nuestras actuaciones cotidianas nos producen miedo y desconfianza, como lo señala Morin (2003, p. 55):

Los seres vivos somos conservadores y esto es central, nos movemos en el supuesto implícito de que el mundo se repite y sigue esencialmente igual. Así lo natural es que conservemos el estilo. Conservemos aquella dinámica relacional en la cual nos sentimos bien, en la cual las cosas que deseamos nos han resultado. Por eso es que es tan difícil el cambio... Para que se abra el espacio del cambio posible, tiene que definir lo que tiene que conservar.

Necesitamos conservar aquello que nos humaniza, actuando desde nuestra conciencia y tomando en cuenta las consecuencias que tienen nuestros actos, siendo libres y flexibles ante nuestras dependencias, obediencias, desconfianzas e inseguridades por lo nuevo; lo diferente que nos interpela: promover aprendizajes verdaderos, con sentido y significado, mediando pedagógicamente los contenidos de los planes de estudio desde el contenido, la forma y los aprendizajes; aplicando la metodología participativa con su bucle procesual en nuestras sesiones de trabajo con el estudiantado; evaluando los aprendizajes con formas éticas, creativas, flexibles e integrales.

Para alcanzar la esencia de la participación con sentido es necesario abrirnos a la integralidad de la vida, conectándonos con nuestro autoconocimiento e inteligencia espiritual, en forma permanente y creativa, para que nuestra conciencia se nutra y sea parte del flujo universal de la vida. 


\section{Referencias}

Abarca, F. (2012). Nuevas visiones para tiempos nuevos: Hacia aprendizajes holistas como derecho de la humanidad. Revista Latinoamericana de Derechos Humanos, 23(2), 61-85. Recuperado de http://www.revistas.una.ac.cr/index.php/derechoshumanos/ article/download/5516/5341

Assman, H. (2002). Placer y ternura en la educación: Hacia una sociedad aprendiente. Madrid, España: Editorial Nancea.

Capra, F. (1994). El punto crucial. Barcelona: Editorial Integral.

Jara, O. (2012). La sistematización de experiencias: Práctica y teoría para otros mundos posibles. San José, Costa Rica: Centro de Estudios y Publicaciones Alforja.

Jara, O. (1987). Las dimensiones de la educación popular. San José, Costa Rica: Centro de Estudios y Publicaciones Alforja.

Leis, R. (1989). El arco y la flechas: Apuntes sobre metodología y práctica transformadora. Panamá: CEASPA.

Morín, E. (2003). El método 5: La humanidad de la humanidad. Madrid: Ediciones Anaya.

Najmanovich, D. (2008). Mirar con nuevos ojos: Nuevos paradigmas en la ciencia y pensamiento complejo. Buenos Aires, Argentina: Editorial Biblos.

Najmanovich, D. (2008). Epistemología para principiantes: Pensamiento científico, metodología de la investigación. Buenos Aires, Argentina: Era Naciente.

Najmanovich, D. (2014). El cambio educativo: Del control disciplinario al encuentro comunitario. Buenos Aires, Argentina: Editorial Biblos.

Najmanovich, D. (2015). Itinerarios de la complejidad: Sitio web: Travesías N. ${ }^{\circ}$ 50. Recuperado de www.denisenajmanovich.com.ar 